more deeply, the authors replaced $\mathrm{Ca}^{2+}$ in the extracellular solution with $\mathrm{Sr}^{2+}$, which results in a more asynchronous release and (aEPSCs) release and allows isolated quantal events to be identified. As before, LRRTM1 KD decreased and SLITRK1 KD increased aEPSCs. These effects were associated with a marked decrease in docked vesicles following LRRTM1 KD and an increase following SLITRK1 KD, a morphological correlate that likely explains the effects of $\mathrm{KD}$ of these proteins on synaptic function.

Conversely, KD of FLRT2 was associated with a marked reduction in CA1 dendritic spine density, but synaptic vesicle distribution was normal. Together with the effects of FLRT2 on synaptic transmission described above, these data show that FLRT2 plays a different role, namely in regulating synaptic current (SC) synapse number.

Temporoammonic (TA) fibres are the other major input to CA1 apical dendrites and terminate in the stratum lacunosum-moleculare (SLM). Selective FLRT2 KD in CA1 corresponding asynchronous EPSCs

neurons followed by stimulation of TA inputs reduced both AMPAR and NMDAR EPSCs and asychronous release - similar to the findings in stratum radiatum - suggesting that FLRT2 controls the number of functional synapses in both stratum radiatum and SLM. LRRTM1 KD did not affect TA synaptic function, which is in line with the low endogenous expression level of LRRTM1 in the SLM. Finally, SLITRK1 KD decreased AMPAR EPSCs but not NMDAR EPSCs, and decreased the amplitude but not the frequency of asynchronous release, suggesting a different role of SLITRK1 at TA inputs, regulating AMPAR density.

Together, these findings reveal that different classes of LRR proteins expressed by CA1 pyramidal cells have distinct roles in regulating synaptic density and function, and that these roles are modular and context dependent.

Sian Lewis

ORIGINAL ARTICLE Schroeder, A. et al. A modular organization of $L R R$ protein-mediated synaptic adhesion molecules defines synapse identity. Neuron https://doi.org/10.1016/j.neuron. 2018.06.026 (2018)

inputs) but very little FOS expression in the ventral and intermediate zones of the spinal cord (where descending inputs arrive). By contrast, CLP290-treated mice or mice treated with a KCC2-expressing virus showed FOS expression distributed more evenly throughout all zones of the spinal cord, similar to uninjured controls. Moreover, cortical stimulation in treated mice evoked larger electromyograph responses in the ankle flexor muscle than in untreated mice. Thus, KCC2 expression or CLP290 improves the efficiency of descending signals to the hindlimb.

The authors reasoned that the downregulation of $\mathrm{KCC} 2$ in spared spinal neurons may make these cells physiologically similar to immature neurons, which, because of their high intracellular levels of chloride, are depolarized by GABA and glycine and therefore hyperexcitable. In line with this assertion, pharmacogenetic reduction of the excitability of inhibitory interneurons around the staggered lesions in injured mice resulted in improved recovery of locomotion.

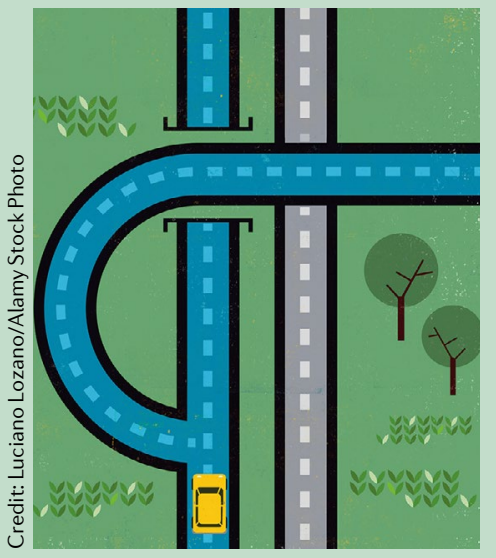

Overall, this study shows that inhibitory spinal cord interneurons spared after incomplete $\mathrm{SCl}$ show reduced levels of $\mathrm{KCC} 2$, resulting in hyperexcitability. Restoring KCC2 function in these neurons reinstates their ability to participate in the spinal relays involved in hindlimb function.

Natasha Bray

ORIGINAL ARTICLE Chen, B. et al. Reactivation of dormant relay pathways in injured spinal cord by KCC2 manipulations. Cell https://doi.org/ 10.1016/j.cell.2018.06.005 (2018)

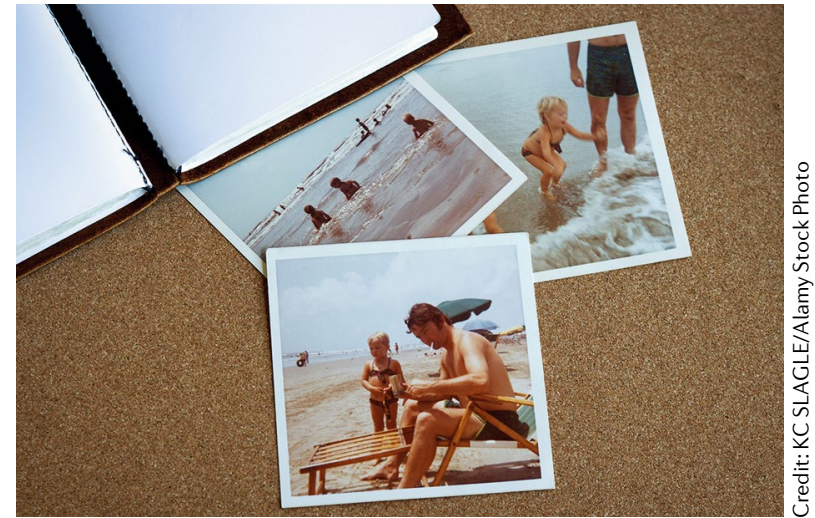

LEARNING AND MEMORY

\section{Bringing back early memories}

As adults, we can usually recall little about events experienced during our early years. This phenomenon has been attributed to the restructuring of hippocampal circuitry in early postnatal life; however, whether the neuronal traces representing infant memories are permanently erased is unknown. Guskjolen et al. now show that infant memory traces are retained in a dormant state in the mouse dentate gyrus (DG) and that stimulation of these 'silent engrams' drives recall of lost memories.

Successful memory retrieval is thought to involve the reactivation of engrams active during memory formation. To investigate the apparent failure of this process in infant memory retrieval, the authors generated transgenic mice in which the neurons active during memory acquisition could be specifically and permanently 'tagged' with a light-activated ion channel, and used this system to investigate engram formation and reactivation following contextual fear conditioning (CFC).

Mice that underwent CFC as adults demonstrated long-lasting memory retention, freezing when re-exposed to the training context for at least 90 days after training. As expected, mice that were trained as infants (at postnatal day 17) exhibited rapid forgetting, with context-induced freezing abolished entirely within 90 days of training. However, when a laser was used to stimulate the DG neurons that had been tagged during training, context-induced freezing was reinstated in these mice, up to 90 days after training.

Next, the authors examined neuronal activity in regions downstream of the DG during light-stimulated recall. During the laser stimulation of the DG, they observed a specific reactivation of neurons that had been tagged during CFC acquisition in the hippocampal CA1 and CA 3 regions and in several cortical regions. Thus, the reactivation of DG engrams activated downstream memory-associated neuronal ensembles.

These findings suggest that the engrams encoding memories that are formed early in life yet rapidly forgotten exist in a silent state in which they cannot be reactivated by natural recall cues.

Katherine Whalley

ORIGINAL ARTICLE Guskjolen, A. et al. Recovery of “lost" infant memories in mice. Curr. Biol. https://doi.org/10.1016/j.cub.2018.05.059 (2018)

FURTHER READING Josselyn, S. A., Köhler, S. \& Frankland, P. W. Finding the engram. Nat. Rev. Neurosci. 16, 521-534 (2015) | Tonegawa, S., Morrissey, M. W. \& Kitamura, T. The role of engram cells in the systems consolidation of memory. Nat. Rev. Neurosci. https://doi.org/10.1038/s41583-018-0031-2 (2018) 